\title{
The Influence of Emulsifier on Rheological and Sensory Properties of Cosmetic Lotions
}

\author{
Tereza Moravkova and Petr Filip \\ Institute of Hydrodynamics, Academy of Sciences of the Czech Republic, 16612 Prague, Czech Republic \\ Correspondence should be addressed to Tereza Moravkova; moravkova@ih.cas.cz
}

Received 14 May 2013; Accepted 9 July 2013

Academic Editor: Steven Suib

Copyright ( 2013 T. Moravkova and P. Filip. This is an open access article distributed under the Creative Commons Attribution License, which permits unrestricted use, distribution, and reproduction in any medium, provided the original work is properly cited.

\begin{abstract}
Three body lotions (water-in-oil type) differing only in the emulsifier content were subjected to rheological measurements and sensory analysis with the aim to obtain coupling between the selected rheological characteristics and sensory factors. Both methods proved different behaviour of the samples caused by the difference in the emulsifying compound. To detect the relations between rheological (application of the power law model) and sensory variables, the four most important characteristics from sensory assessment (ease of pouring from the bottle, ease of spreading on a palm, thickness, and ease of spreading on back of hand) were selected. A close coupling (and hence mutual substitution) was found between consistency parameter $k$ and thickness, $k$ and the ease of pouring from the bottle, and also $k$ and the mean droplet size.
\end{abstract}

\section{Introduction}

Cosmetic lotions are formed by two immiscible liquids with different polarities, and each emulsion is exposed to appearance of various types of instabilities. Creaming, sedimentation, flocculation, Ostwald ripening, coalescence, and phase inversion (Tadros [1]) represent the unwanted changes that should be suppressed. An addition of emulsifier represents an often used method of how to stabilize a structure of emulsions: continuous carrier liquid and the other one are homogeneously distributed in the form of discrete droplets. A presence of emulsifier exhibits two favourable inputs-a decrease in interfacial tension between both liquids and a stabilization of the dispersed phase against coalescence. A choice of emulsifier represents one of the final steps in the process of development of a new cosmetic lotion. Its otherwise inevitable usage $(0.5-5 \%)$ evokes a question on how its application can affect two principal groups of cosmetic-lotion characteristics, namely, the rheological and sensory ones.

Theoretical determination of rheological characteristics is rather complicated. Pal [2] summarizes that emulsion viscosity depends on overall ten entry parameters: shear rate, time, viscosity of continuous and dispersed phase, density of continuous and dispersed phase, particle radius, concentration of particles, thermal energy, and interfacial tension. Danov [3] proposed an analytical expression for the viscosity of dilute emulsions in the presence of emulsifiers. However, cosmetic lotions are generally emulsions with high-water content (70-90\%) and lower viscosity, often o/w (oil-inwater) type. Lotions of w/o (water-in-oil) type are less common. On the other hand, experimental determination of rheological characteristics seems to be in general well elaborated, and they can be used for a prediction of such properties as spreadability or pouring of cosmetic lotion from a bottle.

For evaluation of these properties, sensory analysis is taken as a primary tool differentiating between consumerfriendly and negative attributes of the cosmetic lotions usually used in the form of body lotions or cleansing lotions for face. From the viewpoint of product's sales potential, sensory characteristics occupy a privileged position (Lukic et al. [4]). This is a reason why methodology of sensory evaluation of cosmetic products is intensively investigated. A method presented by Gilbert et al. [5] uses Spectrum Descriptive Analysis to evaluate cosmetic creams with different texturing agents. Spectrum Descriptive Analysis provides an accurate sensory description of products by rating each attribute on absolute 
or universal scales. This method allows the comparison of relative intensities within a product or among products.

From the previous, it is apparent that rheological and sensory properties are coupled, not detached. As stated by Karsheva et al. [6], the rheological behaviour of cosmetic products is one of the most important features not only from technical, but also from the aesthetic point of view. These products are expected not only to be easy to use, but also to meet sensory criteria that will appeal to the customer. Rheological properties are often directly related to the product's sensory attributes and to its performance.

Rheological analysis is quite extended in recent research of cosmetic emulsions, but only some studies include the connection with sensory analysis. Nakagawa and Ueda [7] applied rheological measurements to different cosmetic emulsions and found significant correlation between the rheological measurement results and those of microscopic observations, sensory evaluations, and stability tests. Reeve and Amigoni [8] illustrated the use of rheological characterization for research, development, or quality control through the test of four sun creams with different consistencies. Ibarnescu et al. [9] found a good correlation between rheological parameters and sensory perception of the consumer. Three oil-in-water cosmetic emulsions were studied using simple and oscillatory shear rheological tests. In regard of consumers' demand for natural cosmetics, Karsheva and Georgieva [10] investigated the effect of the plant extracts as well as the influence of the solvent on the product flow properties. Morávková and Stern [11] found statistically significant relationships between rheological and sensory parameters of cosmetic emulsions, concerning both body lotions and face creams.

An important term in sensory analysis of cosmetics is represented by skin feeling. Brummer and Godersky [12] divide skin feeling - the sensations experienced during application-into primary and secondary. The primary skin feeling describes the sensations at the start of the application, and the secondary skin feeling describes the sensations at the end of application. The primary skin feeling correlates with the shear stress at the onset of flow and the dynamic viscosity. The secondary skin feeling correlates with the value of the stationary viscosity for the rate of shear prevailing at the end of application to the skin.

If we take into account the time and cost of sensory analysis and volunteer's subjectivity, it seems that potential use of instrumental measurements correlating with sensory properties is very perspective (Lukic et al. [4]). This approach could eliminate sensory analysis by responsible predictions based on rheological measurements only if a nonsubstantial part of ingredients contained in cosmetic lotions is changed.

The study of influence of a concrete ingredient on both rheological and sensory properties is relatively rare. Lukic et al. [4] studied four water-in-oil creams varying in one emollient component. The samples were submitted to rheological, sensory, and textural characterization. The results indicated that certain alteration restricted to the oil phase induced a change in all investigated characteristics. Obtained correlation between physical measurements and certain sensory attributes confirmed that textural analysis, as a fairly simple measurement, can be used as a surrogate for rheological measurements.

Rheological measurement can be also used for the determination of the optimal content of the active ingredient as applied by Abu-Jdayil et al. [13] who analyzed the Dead Sea salt content in cosmetic emulsions.

The objective of this study is to specify the influence of a small change in the composition of a cosmetic emulsion to its rheological and textural properties. To detect this, three cosmetic emulsions, water-in-oil body lotions, differing in a ratio of emulsifier were prepared. Samples were analyzed by rheological procedures and sensory analysis, and the particle size was evaluated. The results were compared with the ingredients of the sample to assess the role of the emulsifier. Afterwards, a relationship among detected characteristics was specified to find out a connection among the used methods.

\section{Experimental}

2.1. Materials. Three samples of body lotions, denoted as BL1, BL2, and BL3, were prepared. Each sample contains 5 wt.\% of emulsifiers (different for different samples) and 95 wt.\% of other components (identical for all three samples). The composition of the samples is documented in Table 1 (including the suppliers).

The structure of all three body lotions differed only in the composition of an emulsifier. In all three cases, the emulsifier consisted of the three same basic components (see Table 2) but in various weight ratios.

2.2. Devices. A semi-plant mixer BECOMIX RW15 (A. Berents, Stuhr, Germany) was applied for the preparation of the samples. This mixer includes vacuum processing vessel with homogenizer and jacket enabling heating or cooling. It has a batch capacity of 12 litres, with a minimum capacity of 1-2 litres.

Rheological measurements were carried out with a rotational rheometer RheoStress 300 (Thermo Scientific, Karlsruhe, Germany). A cone-and-plate system with sensor C60/ $2^{\circ}$ (cone diameter $60 \mathrm{~mm}$, cone angle $2^{\circ}$ ) was used. In contrast to a plate-and-plate arrangement, the chosen cone-and-plate arrangements respect non-Newtonian course of measured characteristics and, hence, provide more responsible data. All measurements were carried out at $25^{\circ} \mathrm{C}$.

2.3. Samples Preparation. During the emulsification, the mixing intensity corresponded with level 3, and homogenization proceeded 5 minutes at level 5 using the mixer Becomix RW15 (levels 1 and 5 correspond to preset minimum and maximum revolutions, resp.). The water phase was added into the oil phase, and to obtain higher degree of homogenization, temperature was set to $75^{\circ} \mathrm{C}$. To prevent evaporation, thermal sensitive fragrance was added at a reduced temperature of $45^{\circ} \mathrm{C}$. Total amount of one sample attained $10 \mathrm{~kg}$; consequently, it was filled into $250 \mathrm{~mL}$ plastic (PE) bottles and stored at room temperature.

2.4. Methodology. Both rheological and sensory approaches were applied for characterization of the samples. These two attitudes are not strictly separated but just, on the contrary, 
TABLE 1: Ingredients of the samples.

\begin{tabular}{|c|c|c|c|c|c|}
\hline \multirow{2}{*}{ Ingredients } & \multirow{2}{*}{ Ingredients (INCI name) } & \multirow{2}{*}{ Supplier } & \multicolumn{3}{|c|}{ Content [wt.\%] } \\
\hline & & & BL1 & BL2 & BL3 \\
\hline Emulsifiers & \multicolumn{2}{|c|}{ Details in Table 2} & 5 & 5 & 5 \\
\hline Isopropyl myristate & Isopropyl myristate & OLEON Scandinavia N.V & 6 & 6 & 6 \\
\hline Paraffin oil & Paraffinum liquidum & Hansen and Rosenthal & 15 & 15 & 15 \\
\hline Glycerin & Glycerin & Neuber & 3 & 3 & 3 \\
\hline Jojoba oil & Buxus chinensis & Gustav Hess & 0.2 & 0.2 & 0.2 \\
\hline Coenzyme Q10 & Ubiquinone & EISAI, Co., Ltd. & 0.025 & 0.025 & 0.025 \\
\hline Magnesium sulfate & Magnesium sulfate & Brenntag CR & 0.7 & 0.7 & 0.7 \\
\hline Allantoin & Allantoin & Merck & 0.1 & 0.1 & 0.1 \\
\hline D-panthenol & Panthenol & Roche vitamins & 0.5 & 0.5 & 0.5 \\
\hline Nipasept & Methyl, ethyl, propylparaben & Jan Dekker & 0.3 & 0.3 & 0.3 \\
\hline Neolone 950 & Methylisothiazolinone & Rohm and Haas & 0.1 & 0.1 & 0.1 \\
\hline Fragrance & Parfum & Quest Int. & 0.15 & 0.15 & 0.15 \\
\hline Citric acid & Citric acid & Brenntag CR & 0.5 & 0.5 & 0.5 \\
\hline Water, distilled & Aqua & & 68.425 & 68.425 & 68.425 \\
\hline
\end{tabular}

TABLE 2: Emulsifiers contained in the samples.

\begin{tabular}{lccrr}
\hline Component of emulsifier & Component of emulsifier (INCI name) & Supplier & \multicolumn{3}{c}{ Weight ratio } & BL1 & BL2 & BL3 \\
\hline Arlacel 989 & & ICI Surfactants & $3 / 5$ & $2 / 5$ \\
Arlatone T & PEG-7 hydrogenated castor oil & ICI Surfactants & $1 / 5$ & $1 / 5$ \\
Span 60 & PEG-40 sorbitan peroleate & Cognis & $1 / 5$ & $1 / 5$ \\
\hline
\end{tabular}

coupled. Sensory analysis is time and cost demanding; in addition, there is a certain influence of volunteer's subjectivity. Inputs from the sensory analysis can be in some range replaced by the rheological characterization, which represents substantially cheaper and effective solution. To verify this approach, loosely said an acceptable substitution of sensory analysis by the rheological one, for the problem studied (emulsifier alteration) the following steps were carried out:

(i) rheological measurement determining a relation between shear stress and shear rate (the flow curve) in the range of shear rate $10^{-2}-600 \mathrm{~s}^{-1}$. Shear rate continually increased from the minimum to maximum (upcurve) and decreased back to minimum (downcurve). Typical shear rates for pouring from a bottle terminate at approximately $100 \mathrm{~s}^{-1}$, where by coincidence typical shear rates for spreading of lotions start; see, for example, Ward et al. [14];

(ii) sensory evaluation was carried out under standard conditions as specified by the ISO standards (ISO 8589, ISO 8586.1, and ISO 6658). Seventeen trained panellists completed a special questionnaire concerning 11 parameters (including colour, shine, appearance of the surface, feelings during rub-in, and frequency of the use of body lotion). Each parameter was rated on a category scale with predefined descriptive terms. The four most important parameters (the ease of pouring from the bottle, the ease of spreading on a palm and on a back of hand, and thickness) involved, except a category scale, a graphic unstructured one as well. For these four parameters, there were adjusted counter-equivalent rheological qualities measured by the rotational rheometer.

Data processing of both rheological and sensory measurements, and their coupling is presented in Section 3.

\section{Results}

3.1. Rheological Measurement. The flow curves relating shear stress $\tau$ and shear rate $\dot{\gamma}$ were determined. Shear rate continually increased from the minimum $10^{-2} \mathrm{~s}^{-1}$ to $600 \mathrm{~s}^{-1}$ and decreased back to minimum (downcurve). Data for all three body lotions are depicted in Figure 1. The arrows indicate a direction of measurement.

It is apparent that relatively small change in the compositions of BL1, BL2, and BL3 results in substantially different behaviour of flow curves. The change in emulsifier composition is reflected not only in a course of the upward curves but especially in their downward behaviour. This confirms a nonnegligible impact of the emulsifiers used on the overall manifestation of the individual products.

For characterization of flow curves in upward direction, the power-law model was used as follows:

$$
\tau=k \cdot \dot{\gamma}^{n}
$$

where $k$ is the consistency parameter and $n$ is the flow behaviour index. The values of these parameters for all three 
TABle 3: Parameters of the power law model.

\begin{tabular}{lcc}
\hline & $k\left[\mathrm{~Pa} \cdot \mathrm{s}^{n}\right]$ & $n[-]$ \\
\hline BL1 & 28.12 & 0.46 \\
BL2 & 13.90 & 0.52 \\
BL3 & 11.45 & 0.46 \\
\hline
\end{tabular}

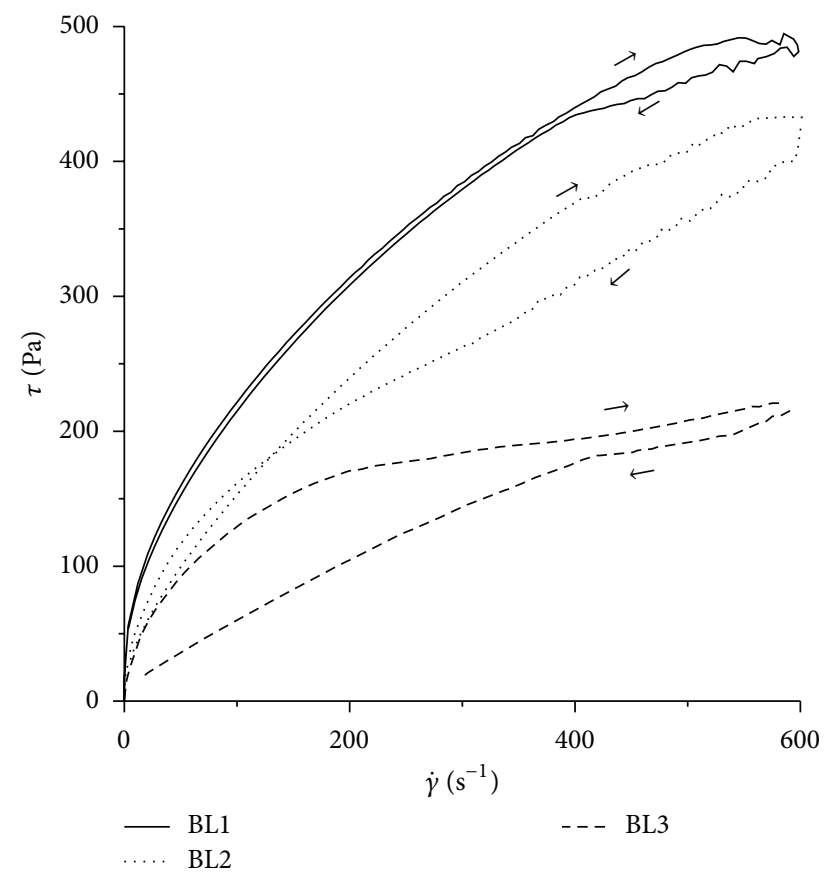

FIGURE 1: The flow curves of BL1, BL2, and BL3 in the range of shear rate $10^{-2}-600 \mathrm{~s}^{-1}$.

body lotions are summarized in Table 3, and the correspondence with the experimental data (upward direction) is presented in Figure 2.

3.2. Sensory Analysis. Table 4 documents the results from the sensory analysis involving all assessed parameters. These parameters were evaluated at a category scale. Each category contained a descriptive term; a list of them is illustrated in Table 4. Trained evaluators signed one or more category on a scale. The descriptions, which were signed most often (for all samples, if not specified) are in the last column of Table 4 .

Ease of spreading is evaluated at two places-on the palm and on the back of hand due to different body temperature. This dual assessment of spreading was proved to be useful (see Morávková and Stern [11]).

The four properties (pouring from the bottle: $\mathrm{P}$, ease of spreading on a palm: SP, thickness: $\mathrm{T}$, and ease of spreading on back of hand: $\mathrm{SH}$ ) range to the most important characterization of the body lotions studied. Their more detailed evaluation is presented in Table 5. The thickness, reflecting in principle viscosity, was evaluated when a body lotion was applied to back of hand using a scale, thin, optimal, and too thick.

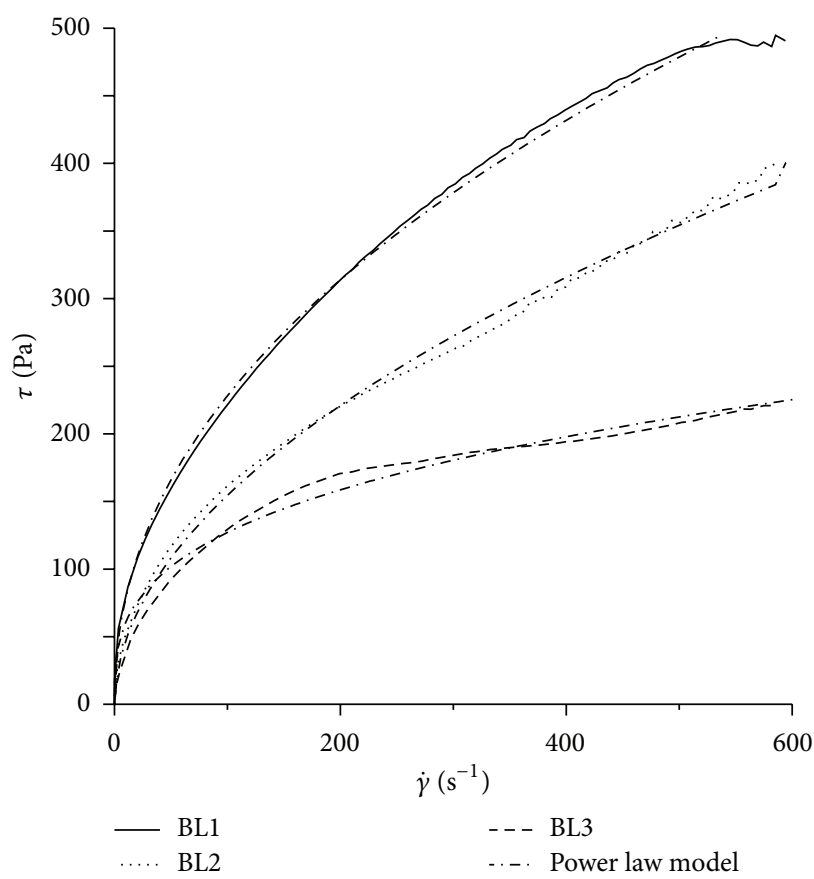

Figure 2: Approximation of the flow curves (upward direction) of $\mathrm{BL1}, \mathrm{BL} 2$, and $\mathrm{BL} 3$ in the range of shear rates $10^{-2}-600 \mathrm{~s}^{-1}$ by the power law model.

Considering ease of spreading (both places), the samples sort BL1, BL2, and BL3 from bad to optimal. The same order settles in the thickness category, BLl being the most thick, BL2 the less, and BL3 the least. Reversely assessment of the pouring from the bottle gives similar values; only lotion BL1 slightly divides with more difficult pouring.

3.3. Basic Morphology. One of the main purposes of the emulsifier is to decrease the interfacial tension and thus help to form the interface between the continuous oil phase and water droplets. This effect should eliminate the breakdown processes in the emulsion. In the presented samples, there is a supposed homogeneity in coating of the interface by an emulsifier as its amount attains $5 \mathrm{wt} \%$. This results in no breakup or tip-dropping of the droplets.

The microstructure of the samples was observed by the laboratory microscope DSML (Leica, Germany) integrated with digital camera. Figure 3 illustrates basic morphology of the body lotion (figure widths attains $80 \mu \mathrm{m}$ ). The received droplet size is documented in Table 6. From here, it follows that the samples BL2 and BL3 are apparently more homogenous than the sample BL1.

3.4. Correlation Relating Rheological and Sensory Measurements. In the following, the linear relations between the sensory variables and rheological parameters obtained by applying the power law model are determined; see Figures 4 and 5. Figure 4 shows consecutively 5 linear approximations of four selected sensory parameters (see Table 5) and droplet size (see Table 6) in dependence on the consistency parameter $k$ (see Table 3 ) for BL1, BL2, and BL3. Figure 5 analogously 
TABLE 4: Results of the sensory analysis.

\begin{tabular}{lll}
\hline Assessed property & Descriptive terms & The most frequent descriptions \\
\hline Frequency of the use of BL & Daily, very often, often, sometimes, rarely, never & Often, sometimes \\
$\begin{array}{l}\text { Pouring from the bottle } \\
\text { Shine of the BL }\end{array}$ & Very easy, easy, difficult, very difficult & Very easy, easy \\
Surface of the BL & Very shiny, shiny, matt, uneven & Very shiny, shiny \\
Ease of spreading on a palm & Smooth, grainy, bubbles, separated oil/water, other inhomogeneity & Smooth, bubbles \\
Thickness & Optimal, satisfactory, bad, very bad & Optimal, satisfactory \\
Ease of spreading on a hand & Thin, optimal, too thick & Optimal \\
Feelings during rub-in & Optimal, satisfactory, bad, very bad & Optimal, satisfactory \\
Fragrance & Cooling, smooth, warm, rough, scratchy, burning, drying out & Cooling, smooth, warm \\
Absorption & Extremely nice, very nice, nice, neutral, unpleasant, unsavory & Very nice, nice \\
Interest in using the BL & Very quick, good, difficult, very difficult & Good, difficult (BL1) \\
\hline
\end{tabular}
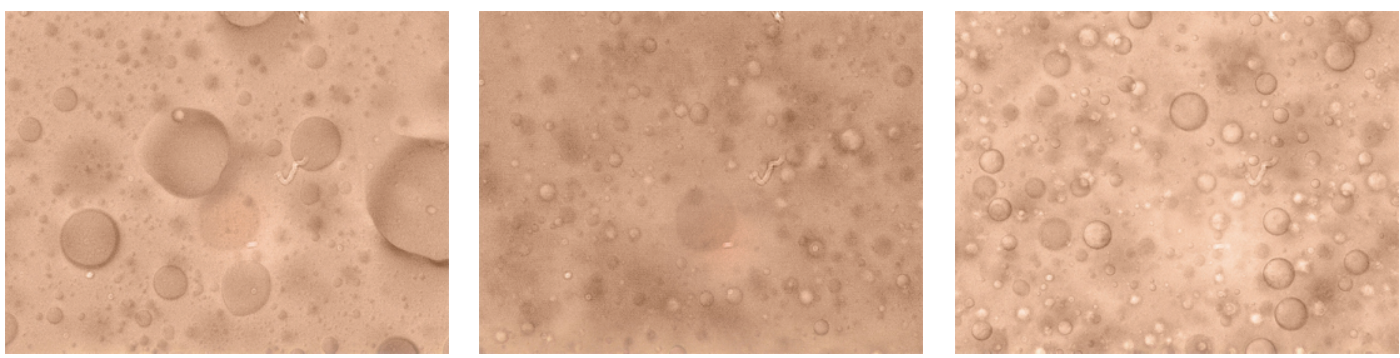

FIGURE 3: Microstructure of samples BL1, BL2, and BL3 (from left to right).

TABLE 5: Results of the sensory analysis, selected parameters (mean values, evaluated at scale $0-100)$.

\begin{tabular}{lcccc}
\hline \multirow{2}{*}{ 100 responds to } & P & SP & T & SH \\
& Very difficult & Optimal & Too much thick & $\begin{array}{c}\text { Optimal } \\
\text { BL1 }\end{array}$ \\
\hline 30 & 65.5 & 54.5 & 66.5 \\
BL2 & 24.5 & 73.5 & 40.5 & 79 \\
BL3 & 24 & 85 & 36.5 & 86 \\
\hline
\end{tabular}

TABLE 6: Measured droplet size.

\begin{tabular}{lcc}
\hline & Droplet size mean value $(\mu \mathrm{m})$ & Standard deviation $(\mu \mathrm{m})$ \\
\hline BL1 & 7.12 & 3.79 \\
BL2 & 5.53 & 1.66 \\
BL3 & 5.36 & 3.27 \\
\hline
\end{tabular}

presents 5 linear approximations in dependence on the flow behaviour index $n$. The individual correlation coefficients are summarized in Table 7. From here, it is apparent that the tightest relations are for the following couples: droplet size, pouring from the bottle; consistency parameter $k$, pouring from the bottle; $k$, thickness; and $k$, droplet size.

As already mentioned above, sensory analysis is time and money consuming, and it is also influenced by volunteer's subjectivity. The above analysis enables eliminating evaluation of the ease of pouring of a bottle and thickness as these properties can be estimated directly from the rheological measurements through the parameter $k$. The parameter $\mathrm{P}$
TABLE 7: Correlation coefficients among rheological and sensory parameters.

\begin{tabular}{lccc}
\hline & $k$ & $n$ & Droplet size \\
\hline $\begin{array}{l}\text { P: pouring the lotion from a } \\
\text { bottle }\end{array}$ & $\mathbf{0 . 9 9 8 1}$ & -0.4731 & $\mathbf{0 . 9 9 9 9}$ \\
$\begin{array}{l}\text { SP: ease of spreading on a hand } \\
\text { T: thickness }\end{array}$ & 0.8822 & 0.0592 & 0.8581 \\
$\begin{array}{l}\text { SH: ease of spreading on a back } \\
\text { of hand }\end{array}$ & 0.9746 & -0.2045 & 0.9625 \\
\begin{tabular}{l} 
Droplet size \\
\hline
\end{tabular} & $\mathbf{0 . 9 9 8 8}$ & -0.4621 & - \\
\hline
\end{tabular}

Statistically significant relations are bold.

(ease of pouring the lotion from a bottle) can be evaluated using the microscope analysis (medium droplet size).

\section{Conclusions}

Three cosmetic lotions, water-in-oil type body lotions, were prepared with different composition of the emulsifier. The other ingredients were identical. All samples were characterized by rheological analysis and sensory profiling. Rheological analysis proved that a small change in emulsifier composition reflects on the behaviour of the lotion; it is illustrated by the differences in the measured flow curves. To characterize the flow properties of the samples, the power law model was applied. The consistency parameter $k$ and the flow behaviour index $n$ were determined. A special questionnaire arranged for the sensory analysis comprised 11 characteristics. Four 


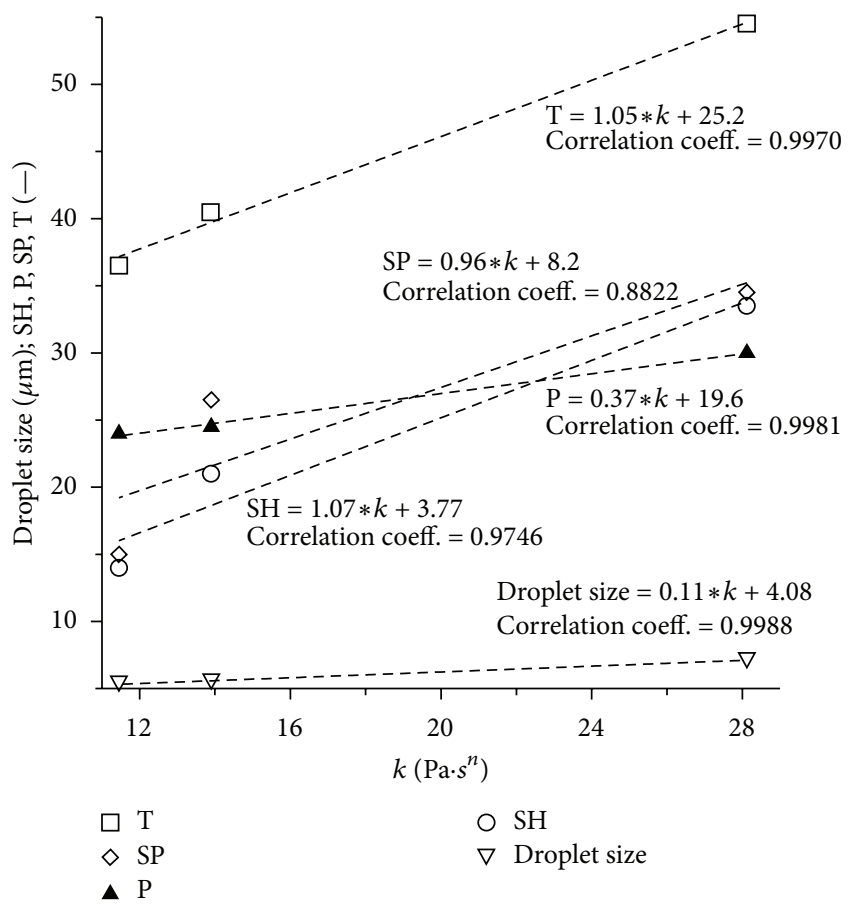

Figure 4: Relationship between sensory parameters (and droplet size) and consistency parameter $k$.

of them, important for consequent coupling with sensory variables, were assessed on a graphic unstructured scale (ease of pouring from the bottle, ease of spreading on a palm, thickness, and ease of spreading on back of hand). The results of sensory analysis confirmed a different manifestation of the samples. These differences were verified also by observing the microstructure of the lotions, specified by the mean droplet size.

To detect the relations between rheological parameters obtained by the power law model and sensory variables from the analysis, the linear approximation was carried out. The correlation coefficients describe the relevance of the relations. Tight relations were found for the following couples: droplet size, pouring from the bottle; $k$, pouring from the bottle; $k$, thickness; and $k$, droplet size. These results enable to recommend the prediction of some sensory parameters (pouring from the bottle and thickness) from the results of rheological measurements. The pouring from the bottle can be also evaluated by the output of the microscope analysis. In contrast to pouring, there is no coupling between the consistency parameter $k$ and spreading tests. This is probably caused by the fact that for pouring from a bottle (shear rates lower than $100 \mathrm{~s}^{-1}$ ) the corresponding flow curves (Figure 2) are more concave in comparison with the section describing a process of spreading (shear rates exceeding $100 \mathrm{~s}^{-1}$ ) where a more "linear" character dominates.

The founded close relationships allow the replacement of time and money consuming sensory assessment by fast instrumental analysis.

A future step in analysing the above studied body lotions will be also based on oscillatory measurements completing

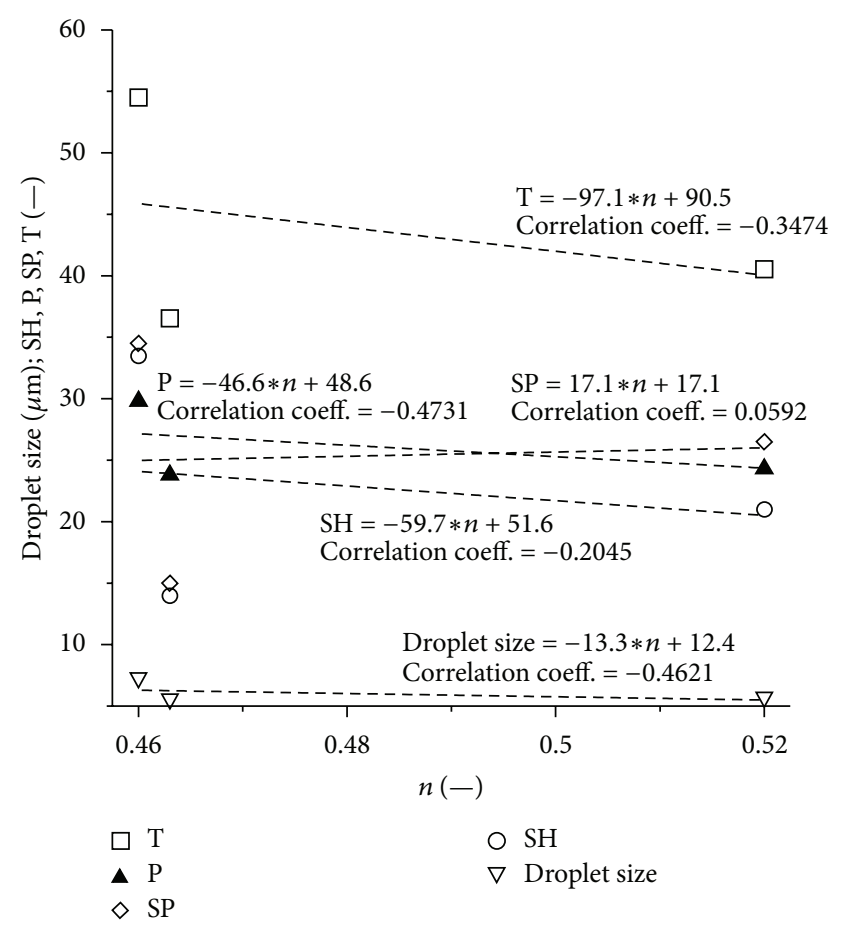

FIGURE 5: Relationship between sensory parameters (and droplet size) and flow behaviour index $n$.

a rheological approach to this topic. This approach provides a very useful means for characterizing textural and sensory properties as recently documented, for example, by Lukic et al. [15].

\section{Acknowledgment}

The authors wish to acknowledge the Grant Agency CR for the financial support of Grant Project no. 103/09/2066.

\section{References}

[1] T. F. Tadros, Rhelogy of Dispersions, Wiley-VCH, Weinheim, Germany, 2010.

[2] R. Pal, "Scaling of relative viscosity of emulsions," Journal of Rheology, vol. 41, no. 1, pp. 141-150, 1997.

[3] K. D. Danov, "On the viscosity of dilute emulsions," Journal of Colloid and Interface Science, vol. 235, no. 1, pp. 144-149, 2001.

[4] M. Lukic, I. Jaksic, V. Krstonosic, N. Cekic, and S. Savic, "A combined approach in characterization of an effective w/o hand cream: the influence of emollient on textural, sensorial and in vivo skin performance," International Journal of Cosmetic Science, vol. 34, no. 2, pp. 140-149, 2012.

[5] L. Gilbert, C. Picard, G. Savary, and M. Grisel, "Impact of polymers on texture properties of cosmetic emulsions: a methodological approach," Journal of Sensory Studies, vol. 27, pp. 392402, 2012.

[6] M. Karsheva, S. Georgieva, and S. Alexandrova, "Rheological behavior of sun protection compositions during formulation," Korean Journal of Chemical Engineering, vol. 29, no. 12, pp. 18061811, 2012. 
[7] Y. Nakagawa and T. Ueda, "The application of rheology in the development of unique cosmetics," Nihon Reoroji Gakkaishi, vol. 38, no. 4-5, pp. 175-180, 2010.

[8] P. Reeve and S. Amigoni, "Rheology: a precious tool for cosmetic formulation," Actualite Chimique, no. 323-324, pp. 89-98, 2008.

[9] C. Ibǎnescu, M. Danu, A. Nanu, M. Lungu, and B. C. Simionescu, "Stability of disperse systems estimated using rheological oscillatory shear tests," Revue Roumaine de Chimie, vol. 55, no. 11-12, pp. 933-940, 2010.

[10] M. Karsheva and S. Georgieva, "Flow properties of phytocosmetic formulations. Effect of plant extracts and thickeners," Comptes Rendus de L'Academie Bulgare des Sciences, vol. 63, no. 12, pp. 1725-1732, 2010.

[11] T. Morávková and P. Stern, "Rheological and textural properties of cosmetic emulsions," Applied Rheology, vol. 21, no. 3, Article ID 35200, 6 pages, 2011.

[12] R. Brummer and S. Godersky, "Rheological studies to objectify sensations occurring when cosmetic emulsions are applied to the skin," Colloids and Surfaces A, vol. 152, no. 1-2, pp. 89-94, 1999.

[13] B. Abu-Jdayil, H. A. Mohameed, and A. Bsoul, "Determination of optimal dead sea salt content in a cosmetic emulsion using rheology and stability measurements," Journal of Cosmetic Science, vol. 59, no. 1, pp. 1-14, 2008.

[14] J. B. Ward, J. F. Kinney, and H. Y. Saad, "Application of rheological studies to product formulation, stability, and processing problems," Journal of the Society of Cosmetic Chemists of Japan, vol. 25, no. 8, pp. 437-454, 1974.

[15] M. Lukic, I. Jaksic, V. Krstonosic, L. Dokic, and S. Savic, "Effect of small change in oil phase composition on rheological and textural properties of w/o emulsion," Journal of Texture Studies, vol. 44, pp. 34-44, 2013. 

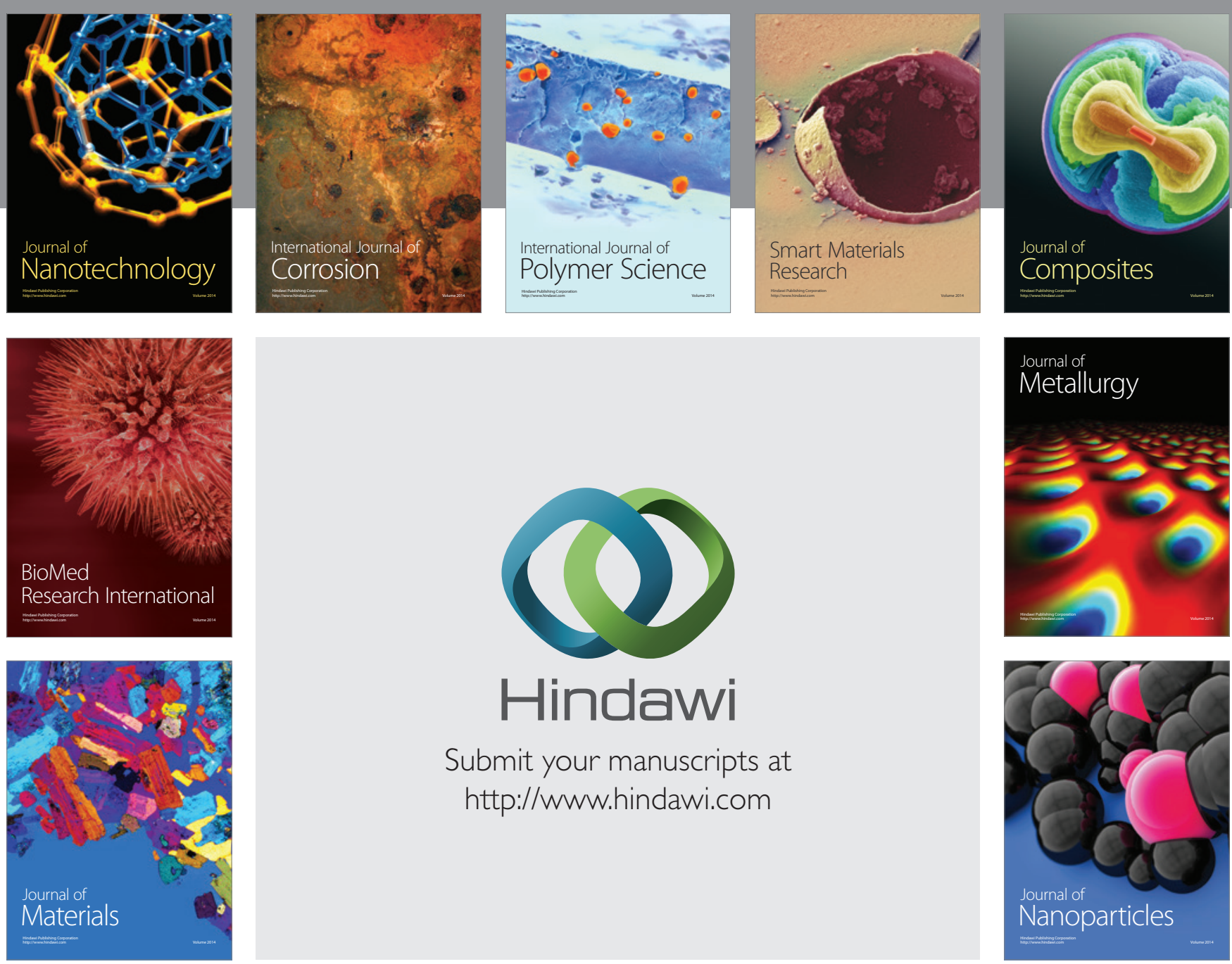

Submit your manuscripts at http://www.hindawi.com
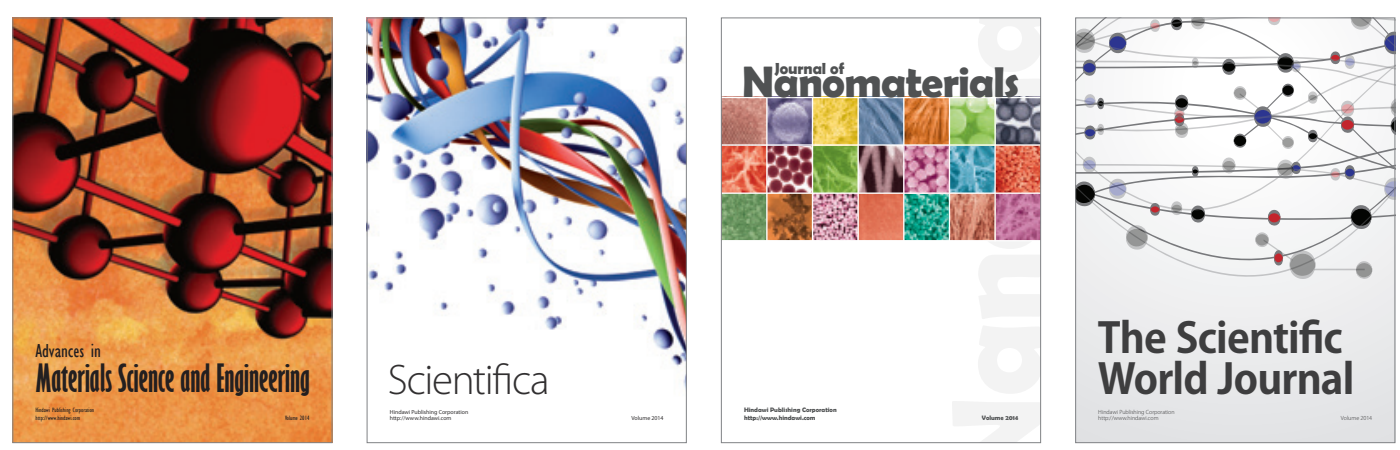

\section{The Scientific World Journal}
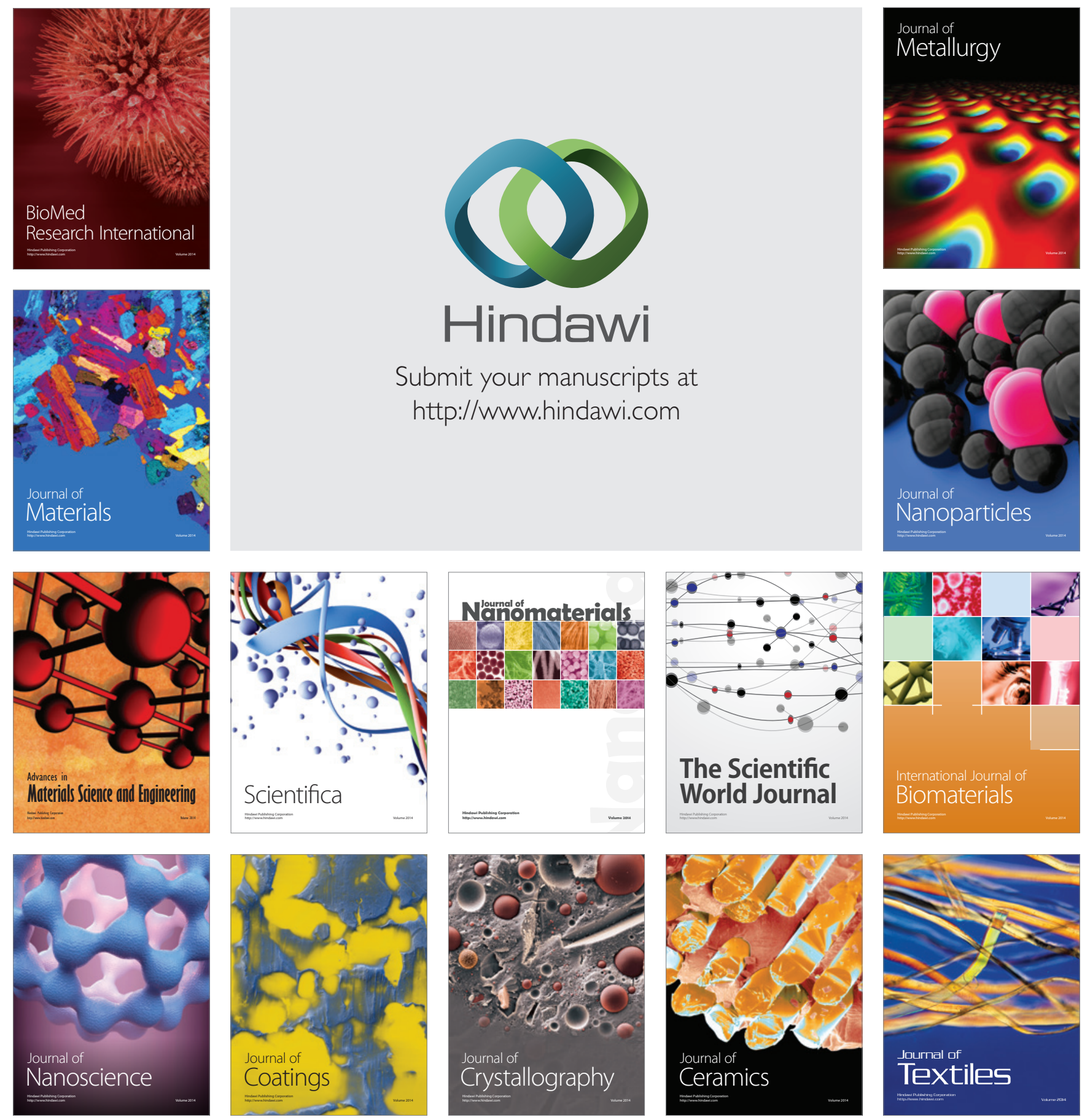